\title{
Low-loss single-mode hybrid-lattice hollow-core photonic-crystal fibre
}

\author{
Foued Amrani ${ }^{1,2}$, Jonas H. Osório (10), Frédéric Delahaye ${ }^{1,2}$, Fabio Giovanardi ${ }^{3}$, Luca Vincetti (3) , Benoît Debord ${ }^{1,2}$, \\ Frédéric Gérôme ${ }^{1,2}$ and Fetah Benabid ${ }^{1,2}$
}

\begin{abstract}
Remarkable recent demonstrations of ultra-low-loss inhibited-coupling (IC) hollow-core photonic-crystal fibres (HCPCFs) established them as serious candidates for next-generation long-haul fibre optics systems. A hindrance to this prospect and also to short-haul applications such as micromachining, where stable and high-quality beam delivery is needed, is the difficulty in designing and fabricating an IC-guiding fibre that combines ultra-low loss, truly robust single-modeness, and polarisation-maintaining operation. The design solutions proposed to date require a trade-off between low loss and truly single-modeness. Here, we propose a novel IC-HCPCF for achieving low-loss and effective single-mode operation. The fibre is endowed with a hybrid cladding composed of a Kagome-tubular lattice (HKT). This new concept of a microstructured cladding allows us to significantly reduce the confinement loss and, at the same time, preserve truly robust single-mode operation. Experimental results show an HKT-IC-HCPCF with a minimum loss of $1.6 \mathrm{~dB} / \mathrm{km}$ at $1050 \mathrm{~nm}$ and a higher-order mode extinction ratio as high as $47.0 \mathrm{~dB}$ for a $10 \mathrm{~m}$ long fibre. The robustness of the fibre single-modeness is tested by moving the fibre and varying the coupling conditions. The design proposed herein opens a new route for the development of HCPCFs that combine robust ultra-low-loss transmission and single-mode beam delivery and provides new insight into IC guidance.
\end{abstract}

\section{Introduction}

Due to their excellent performance as a platform for the study of fundamental physics and for addressing applied problems in photonics, hollow-core photonic-crystal fibres (HCPCFs) continue to be the subject of intense research since their theoretical proposal in $1995^{1}$. These interests are driven by the accomplishment of outstanding results in science, such as atom optics ${ }^{2}$ and gas-based nonlinear optics ${ }^{3}$, and in industry, such as ultra-shortpulse and high-energy laser beam delivery ${ }^{4}$. Furthermore, the physics of HCPCF optical guidance is still a fascinating and ongoing topic of research ${ }^{5}$.

Today, we distinguish two types of HCPCF: one type that guides via a photonic bandgap $(\mathrm{PBG})^{1}$ and a second

\footnotetext{
Correspondence: Fetah Benabid (f.benabid@xlim.fr)

'GPPMM Group, XLIM Institute, CNRS UMR 7252, University of Limoges, Limoges 87060, France

${ }^{2}$ GLOphotonics, 123 Avenue Albert Thomas, Limoges 87060, France

Full list of author information is available at the end of the article
}

type that guides via the mechanism of inhibited-coupling (IC) optical guidance ${ }^{6}$. In PBG fibres, light is guided because the fibre microstructure is designed such that there is no cladding mode to which the core mode can couple. Conversely, in IC fibres, although there is no bandgap in the cladding frequency-effective refractive index space, the coupling of the core mode to the cladding is strongly inhibited due to a low spatial overlap between the core and cladding mode field distribution, and to a strong mismatch between the transverse spatial phases of these modes ${ }^{7}$. These principles provided conceptual tools to introduce the hypocycloid core contour (i.e., negative curvature) concept $^{8,9}$, which enabled a dramatic enhancement in the light confinement of these fibres, as exemplified by IC-guiding hypocycloid core-contour Kagome lattice HCPCFs and single-ring tubular-lattice (SR-TL) $\mathrm{HCPCFs}^{10}$. Experimental illustrations of the negative curvature core-contour impact are the reduction in loss figures in Kagome HC-PCFs down to $8.5 \mathrm{~dB} / \mathrm{km}$ at

\section{(c) The Author(s) 2021}

\footnotetext{
(c) Open Access This article is licensed under a Creative Commons Attribution 4.0 International License, which permits use, sharing, adaptation, distribution and reproduction in any medium or format, as long as you give appropriate credit to the original author(s) and the source, provide a link to the Creative Commons license, and indicate if changes were made. The images or other third party material in this article are included in the article's Creative Commons license, unless indicated otherwise in a credit line to the material. If material is not included in the article's Creative Commons license and your intended use is not permitted by statutory regulation or exceeds the permitted use, you will need to obtain permission directly from the copyright holder. To view a copy of this license, visit http://creativecommons.org/licenses/by/4.0/.
} 
$1030 \mathrm{~nm}^{11}$, which is considerably lower than the $\mathrm{dB} / \mathrm{m}$ loss level reported in the first Kagome fibre ${ }^{12}$, and the realisation of optimised SR-TL HCPCFs with a transmission loss as low as $7.7 \mathrm{~dB} / \mathrm{km}$ at $780 \mathrm{~nm}^{7}$ and $13.8 \mathrm{~dB} /$ $\mathrm{km}$ at $539 \mathrm{~nm}^{13}$. Among the noteworthy conclusions of this effort is the fact that for wavelengths shorter than $\sim 1 \mu \mathrm{m}$, the transmission loss is no longer limited by the cladding design. Instead, the surface-roughness-induced scattering loss (SSL) is the limiting factor. On the other hand, for wavelengths longer than $\sim 1 \mu \mathrm{m}$, the confinement loss $(\mathrm{CL})$, and hence the cladding design, remains the limiting factor of the IC-HCPCF transmission performance ${ }^{7}$. Very recently, new negative curvature cladding designs were introduced, and lower $\mathrm{CL}$ values than those of Kagome-HCPCFs and SR-TL HCPCFs were demonstrated. Very promising transmission loss figures were reported, as exemplified by the figures of $2 \mathrm{~dB} / \mathrm{km}$ at $1512 \mathrm{~nm}$ for conjoined tubular cladding $\mathrm{HC}-\mathrm{PCFs}^{14}$ and $0.28 \mathrm{~dB} / \mathrm{km}$ at $1550 \mathrm{~nm}$ for nested ${ }^{15}$ tubular cladding HCPCFs. Table 1 summarises the measured loss figures in representative HCPCFs.

While the recent decreasing trend in the IC-HCPCF loss figures is remarkable, the current challenge in the field is to design and fabricate an IC-guiding fibre that combines ultralow loss, truly single-modeness, and polarisation-maintaining operation, especially at wavelengths much shorter than $1550 \mathrm{~nm}$, e.g., around $1 \mu \mathrm{m}$. To illustrate the difficulty in combining only singlemodeness and ultralow loss in IC-guiding fibres at $\sim 1 \mu \mathrm{m}$, we recall the following. Rigorously speaking, in

Table 1 Summary of the measured transmission loss figures and HOM (higher-order mode) extinction in representative HCPCFs

\begin{tabular}{|c|c|c|}
\hline Fibre design & $\begin{array}{l}\text { Minimum loss and } \\
\text { working wavelength }\end{array}$ & $\begin{array}{l}\text { Typical HOM } \\
\text { extinction }\end{array}$ \\
\hline Kagome lattice & $8.5 \mathrm{~dB} / \mathrm{km}$ at $1030 \mathrm{~nm}^{11}$ & $20.2 \mathrm{~dB}$ for $5 \mathrm{~m}^{11}$ \\
\hline \multirow{6}{*}{$\begin{array}{l}\text { Single-ring tubular } \\
\text { lattice (SR-TL) }\end{array}$} & $500 \mathrm{~dB} / \mathrm{km}$ at $1000 \mathrm{~nm}$ & \multirow{6}{*}{$\begin{array}{l}22.4 \mathrm{~dB} \text { for } 15 \mathrm{~m} \\
\text { (8-tube lattice) }\end{array}$} \\
\hline & $(6 \text {-tube lattice })^{19}$ & \\
\hline & $7.7 \mathrm{~dB} / \mathrm{km}$ at $780 \mathrm{~nm}$ & \\
\hline & (8-tube lattice) $)^{7}$ & \\
\hline & $13.8 \mathrm{~dB} / \mathrm{km}$ at $539 \mathrm{~nm}$ & \\
\hline & (9-tube lattice) $)^{13}$ & \\
\hline Conjoined tube lattice & $2 \mathrm{~dB} / \mathrm{km}$ at $1512 \mathrm{~nm}^{14}$ & $>27 \mathrm{~dB}$ for $15 \mathrm{~m}^{14}$ \\
\hline \multirow[t]{2}{*}{ Nested tube lattice } & $0.28 \mathrm{~dB} / \mathrm{km}$ at $1550 \mathrm{~nm}^{15}$ & \multirow[t]{2}{*}{$\sim 25 \mathrm{~dB}$ for $10 \mathrm{~m}^{29}$} \\
\hline & $2.5 \mathrm{~dB} / \mathrm{km}$ at $1000 \mathrm{~nm}^{20}$ & \\
\hline $\begin{array}{l}\text { Hybrid Kagome-tubular } \\
\text { (HKT) lattice }\end{array}$ & $\begin{array}{l}1.6 \mathrm{~dB} / \mathrm{km} \text { at } 1050 \mathrm{~nm} \\
\text { (this work) }\end{array}$ & $\begin{array}{l}47.0 \mathrm{~dB} \text { for } 10 \mathrm{~m} \\
\text { (this work) }\end{array}$ \\
\hline
\end{tabular}

IC-guiding fibres, single-modeness is impossible because of the ubiquitous presence of higher-order modes in the fibre core. However, one can get closer to a truly singlemode operation if the loss extinction ratio between the lowest loss mode (typically, the core fundamental mode) and the second-lowest loss mode is sufficiently high. It was successfully achieved in six-tube SR-TL HCPCFs thanks to the adequate ratio between the core and lattice tube diameters, $D_{\text {tubes }} / D_{\text {core }}$, calculated to be 0.68 , which provides effective refractive index matching between the $\mathrm{LP}_{11}$-like modes of the core (which are usually the main contaminating higher-order mode in the fibre modal content) and the fundamental $\mathrm{LP}_{01}$-like mode of the lattice tubes ${ }^{16-18}$. The inconvenience of this approach, however, is that the CL of the core fundamental mode is considerably high because of the small core diameter. For example, Gao et al. measured loss values of approximately $500 \mathrm{~dB} / \mathrm{km}$ for wavelengths of approximately $1 \mu \mathrm{m}$ with six-tube SR-TL HCPCFs ${ }^{19}$. On the other hand, the minimum loss figure reported in the literature when using IC-guiding fibres of approximately $1 \mu \mathrm{m}$ is $2.5 \mathrm{~dB} / \mathrm{km}$, which was obtained by using a nested tubular cladding $\mathrm{HCPCF}^{20}$. However, the ratio between the loss values of the fundamental and $\mathrm{LP}_{11}$-like modes in nested fibres is typically lower than $20 \mathrm{~dB}^{15}$, which is considerably inferior to the ratio achieved in six-tubelattice SR-TL HCPCFs, which can be higher than 30 $\mathrm{dB}^{18}$. While this ratio is sufficient to achieve effective single-mode operation under static conditions by suitable input light coupling, it becomes problematic to ensure single-modeness in conditions where the fibre is under constant motion and bending. Table 1 summarises the measured higher-order mode extinction levels in representative HCPCFs.

Here, we present the design and fabrication of a novel HCPCF structure that combines single-mode operation and ultralow CL. The cladding design exhibits a hybrid lattice made of Kagome and tubular cladding lattices. The utilisation of two IC claddings allows a decrease in CL while keeping the fibre core demarcated by a six-tubetubular lattice for effective single-mode operation. As shown in the following, a minimum loss figure of $1.6 \mathrm{~dB} /$ $\mathrm{km}$ at $1050 \mathrm{~nm}$ can be experimentally achieved by using this fibre design. Moreover, the modal content of the fibre is measured by using a spatially and spectrally $\left(S^{2}\right)$ imaging technique ${ }^{21}$. The higher-order mode contributions are measured to have a maximum extinction ratio as low as $47.0 \mathrm{~dB}$ for a $10 \mathrm{~m}$ long fibre under optimised coupling conditions. Finally, the robustness of the fibre singlemode character is verified by inspecting the output mode, while the fibre is moving and by changing the coupling conditions. We believe that the fibre design proposed herein provides a new avenue for obtaining low-loss single-mode HCPCFs. 
a

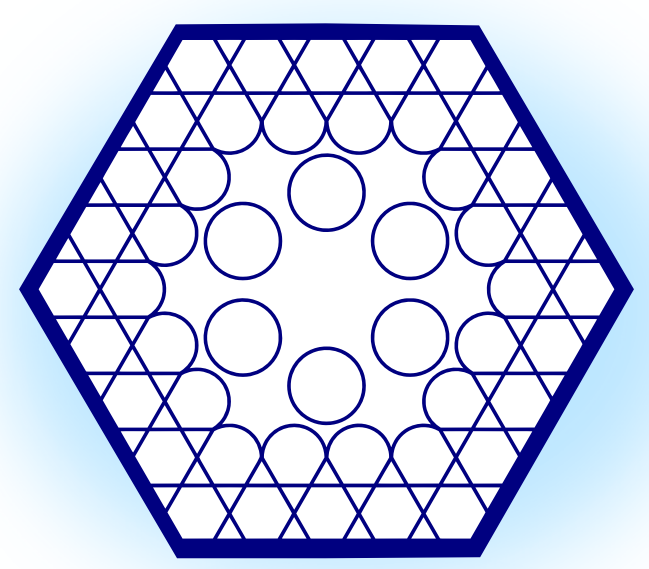

b

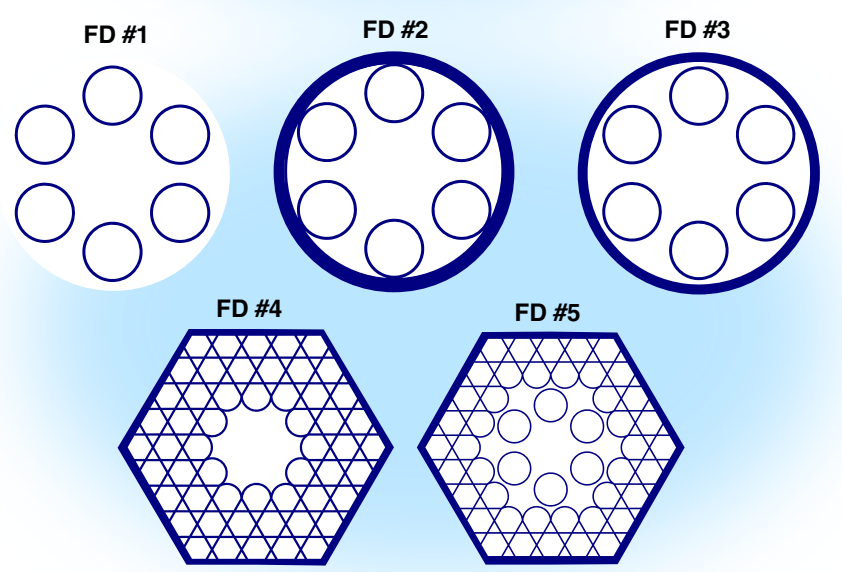

C
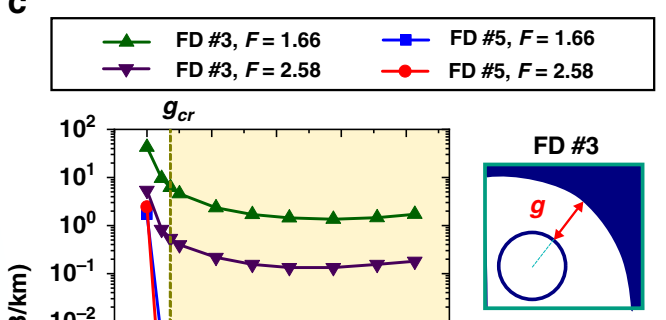

产

$10^{-3}$

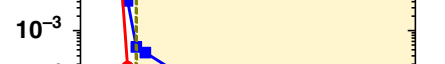

FD \#5

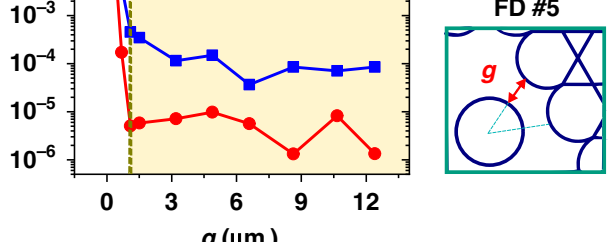

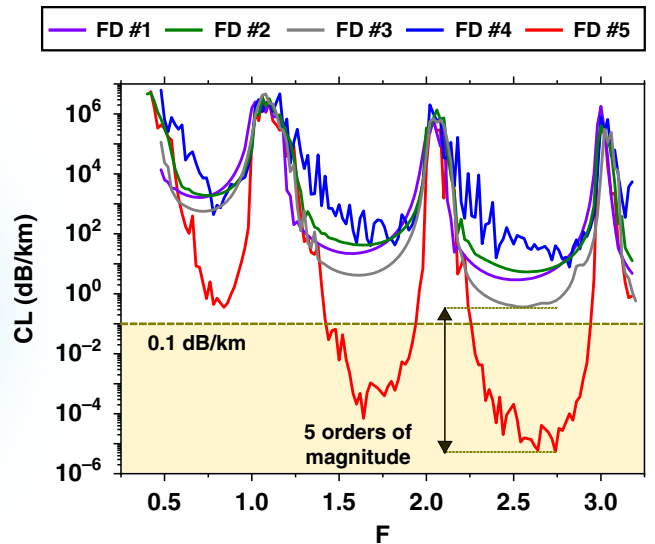

Fig. 1 Fiber design rationale. a Schematic diagram of the hybrid Kagome-tubular HCPCF. $\mathbf{b}$ CL simulation results for different fibre designs (FD). FD \#1: jacketless tubular lattice; FD \#2: jacketed tubular lattice; FD \#3: tubular lattice design with a spacing between the tubes and the silica jacket; FD \#4: Kagome lattice; FD \#5: hybrid lattice. $\mathbf{c ~ C L}$ as a function of the distance $(\mathbf{g})$ between the lattice tubes and the silica jacket (for FD \#3) or between the lattice tubes and Kagome lattice (for FD \#5) at selected normalised frequency values ( $F$ )

\section{Results}

\section{Design rationale}

Figure 1 summarises the proposed fibre structure, the hybrid Kagome-tubular lattice (HKT) HCPCF, and its design rationale. Figure 1a shows the fibre transverse geometrical microstructure. It is endowed with a twolattice cladding. The inner cladding consists of a lattice of six tubes, which demarks the fibre core. The six-tube lattice is chosen to attain effective single-mode operation via resonant filtering of the $\mathrm{LP}_{11}$ mode ${ }^{16-18}$. Additionally, this inner cladding stands out because of the absence of connection nodes, which favours the IC guidance ${ }^{7}$. The outer cladding of the HKT-HCPCF comprises a Kagome lattice structure in which the tubular lattice is embedded.
As will be demonstrated in the following, the association of two IC claddings significantly enhances the light confinement in the core and reduces the fibre CL expressively.

We start our analysis by considering an ideal version of the HKT-HCPCF in which the tubular and Kagome lattices are not physically connected. Even though the ideal structure is unrealistic, it allows exploring the potential of the novel design proposed herein to provide ultralow loss, and to deepen our understanding of the enhancement of the confinement capabilities when combining two IC cladding architectures. Moreover, the ideal version of the fibre acts as a pedagogical and pre-design tool that reveals the most important design elements and physical aspects 
of the proposed fibre architecture, namely, the importance of having a suitable spacing between the inner and outer claddings, the effect of the number of Kagome cladding layers, and the location of the leakage channels in the structure. The presentation of a realisable version of the HKT-HCPCF will follow the study of the ideal version of it.

The fibre design is driven by the guiding principles of the IC guidance mechanism ${ }^{6,7}$. The inner cladding is made with six isolated tubes because doing so ensures single-mode operation and is void of connecting nodes. The surrounding cladding is made by using another IC cladding, i.e., the Kagome lattice, to reduce tunnelling leakage. Furthermore, the CL induced by the nodes and sharp bends in the outer cladding is minimised, because of their larger distance from the fibre core.

Figure $1 \mathrm{~b}$ shows the cross-sections of the fibre designs (FDs), we study herein (lhs) and their respective simulated $\mathrm{CL}$ as a function of the normalised frequency values, $F=$ $(2 t / \lambda) \sqrt{n_{\mathrm{g}}^{2}-1}(r h s)$. Here, $t$ and $n_{\mathrm{g}}$ represent the thickness and refractive index of the cladding structure, respectively, and $\lambda$ is the wavelength. In the CL calculation, all the fibres are taken to have the same core inner diameter $(35 \mu \mathrm{m}), t(1100 \mathrm{~nm})$, and $n_{\mathrm{g}}(1.45)$. The first design (FD \#1, purple line) consists of a jacketless 6-tube lattice structure whose minimum $\mathrm{CL}$ values are approximately $1650 \mathrm{~dB} / \mathrm{km}, 20 \mathrm{~dB} / \mathrm{km}$, and $3 \mathrm{~dB} / \mathrm{km}$ in the fundamental, first-order, and second-order transmission bands, respectively. The second design (FD \#2, green line) reproduces a 6-tube lattice SR-TL HCPCF. The results show that jacketing the suspended tubes impacts the CL spectrum mildly, with an increase in the above loss figures to approximately $1900 \mathrm{~dB} / \mathrm{km}, 40 \mathrm{~dB} / \mathrm{km}$, and $5.5 \mathrm{~dB} / \mathrm{km}$, respectively. FD \#3 (grey line), in turn, is a modified version of the tubular lattice design with tubes that are fully isolated. The spacing between the lattice tubes and the silica jacket is set to $1.59 \mu \mathrm{m}$. In this idealised design, the existence of a gap between the tubes and the jacket affects the CL values, which are found to be lower than those calculated for FD \#1 and FD \#2.

The blue line in Fig. 1b shows the CL for a Kagome lattice HCPCF, identified as FD \#4. For this design, the minimum $\mathrm{CL}$ figures are approximately $440 \mathrm{~dB} / \mathrm{km}$, $50 \mathrm{~dB} / \mathrm{km}$, and $30 \mathrm{~dB} / \mathrm{km}$ in the fundamental, first-order, and second-order transmission bands, respectively. We note for this fibre the oscillating structure in the $\mathrm{CL}$ spectrum due to connecting struts or nodes ${ }^{6}$, which can either locally increase or decrease the CL compared to FD \#1 and FD \#2.

The HKT-HCPCF we propose herein is identified in Fig. $1 \mathrm{~b}$ as FD \#5. The red line in Fig. 1b shows the ideal case of this structure. In this situation, there is no physical connection between the tubular and Kagome lattices, and the spacing between the lattices is set to $1.59 \mu \mathrm{m}$. Although this is not a feasible fibre design, it communicates the potential of this novel design to achieve impressively low $\mathrm{CL}$ values-as low as $0.35 \mathrm{~dB} / \mathrm{km}, 7 \times 10^{-5} \mathrm{~dB} / \mathrm{km}$ and $8.6 \times 10^{-6} \mathrm{~dB} / \mathrm{km}$ for wavelengths within the fundamental, first-order, and second-order transmission bands, respectively. It is noteworthy that the two latter values for this ideal structure are well below the current attenuation level of solid-core fibres, represented by the yellow dashed line in Fig. $1 b^{22}$. Additionally, there is an expressive difference of five orders of magnitude between the CL of FD \#5 and those calculated for the other designs, as emphasised by the black arrow in Fig. 1b. Particularly, a comparison between the results of FD \#3 and FD \#5 allows ascribing the $\mathrm{CL}$ reduction to the replacement of the homogeneous silica jacket by the Kagome microstructure.

Figure 1c shows the evolution, with respect to the spacing between the inner and outer claddings in FD \#3 and FD \#5 ( $g$, see inset of Fig. 1c), of the minimum CL in the 1st and 2nd transmission bands. The results for FD \#5 readily show that, for a significant drop in CL, $g$ must be larger than a critical value $g_{\mathrm{cr}}$. When $g$ is varied from 0.68 to $1.51 \mu \mathrm{m}$, the CL drops from $4.1 \times 10^{-3} \mathrm{~dB} / \mathrm{km}$ to $3.5 \times$ $10^{-4} \mathrm{~dB} / \mathrm{km}$ at $F=1.66$ and from $1.7 \times 10^{-4} \mathrm{~dB} / \mathrm{km}$ to $5.8 \times 10^{-6} \mathrm{~dB} / \mathrm{km}$ at $F=2.58$. The case where $g=0$ is also shown in Fig. 1c. For this case, the CL values are much higher, as high as $1.7 \mathrm{~dB} / \mathrm{km}$ at $F=1.66$ and as high as $2.4 \mathrm{~dB} / \mathrm{km}$ at $F=2.58$. In these simulations, the alteration in the $g$ values is achieved by adequately enlarging the Kagome lattice pitch so that the core diameter $\left(D_{\text {core }}=\right.$ $35 \mu \mathrm{m})$, the distance between the cladding tubes $(\delta=$ $6.5 \mu \mathrm{m})$, and the thickness of the tubes and Kagome lattices $\left(t_{\text {tubes }}=t_{\text {kago }}=1100 \mathrm{~nm}\right)$ can be maintained. Based on the data presented in Fig. 1c, the critical $g$ value $\left(g_{\text {cr }}\right)$ is found to be approximately $1.5 \mu \mathrm{m}$ for attaining the dramatic reduction in the CL in the HKT-HCPCF design compared to that in the usual Kagome and tubular fibre designs. It is noteworthy that the results of our numerical study (not shown) also indicate that the azimuthal position of the inner cladding relative to the outer cladding affects the CL. The results for FD \#3, in turn, also teach that increasing $g$ entails a reduction in the CL values. However, as the confining power of the homogeneous silica jacket is low, the decreasing trend of the CL values is not as extreme as in FD \#5.

Moreover, the concept of CL reduction by associating two IC lattices can be expanded to other IC cladding architectures, such as those with inner claddings made with nested tubes or conjoined tubes (see Supplemental Material for further information). The CL obtained with both the nested tube and conjoined tube inner claddings is comparable with that of the tubular lattice, but they exhibit a structured loss spectrum because of the presence of connecting nodes. Furthermore, it is worth-mentioning that it is particularly challenging to fulfil the required 
phase matching between the $\mathrm{LP}_{11}$ core mode and $\mathrm{LP}_{01}$ inner cladding air modes for single-modeness when using nested tube lattices. Additionally, extending the hybrid design to different outer cladding structures other than the IC structures is a plausible direction. For example, one can propose a different configuration in which the outer Kagome lattice is replaced with a PBG cladding. Indeed, the concept of the association of two claddings to enhance light confinement remains valid. However, further investigation is needed if this approach is chosen since care must be taken in the design and fabrication of the interface between the inner and outer claddings, and in the choice of their respective structural dimensions.

To provide physical insight into the increase in confinement power in the HKT lattice cladding, we recall that, in contrast to PBG fibres, whose loss figures monotonically decrease with an increasing number of cladding layers ${ }^{23}$, the CL in Kagome fibres does not display the same behaviour. Instead, the minimum CL in Kagome fibres is reached for an optimum number of layers, which stems from a trade-off between the cladding structure confining power and the growth in the cladding density of photonic states ${ }^{6}$. Indeed, although adding cladding layers improves the confinement of the mode in the core, it concurrently creates additional cladding modes to which the core mode is weakly coupled, thus entailing further CL. Therefore, the optimum CL in Kagome lattices is accomplished by adequately considering the compromise between the number of photonic states in the cladding and the confining power of the structure. This feature is investigated and corroborated in a systematic study summarised in Figs. 2 and 3.

Figure 2 shows the $\mathrm{CL}$ when considering a 6-tube lattice structure and sequentially adding the Kagome structure around the tubular cladding layer by layer. For simplicity, we define a parameter $\xi$ as the ratio between the thickness of the considered outer cladding in the simulations and the Kagome cladding pitch. In our analysis, $\xi$ is varied from 0 (no Kagome cladding) to 2 (Kagome lattice composed of two rings of tubes), and the calculated $\mathrm{CL}$ values are presented in Fig. 2a. Figure 2b shows the $\mathrm{CL}$ values for two representative normalised frequency values $(F=1.66$ and $F=2.58$ ). The effect of adding the Kagome cladding on the CL drop rate is extremely drastic between $\xi=0$ and $\xi=0.5$ and gentler for $\xi>1$. At $F=1.66$, the $\mathrm{CL}$ is calculated to be $17.4 \mathrm{~dB} / \mathrm{km}$ when $\xi=0$ and $6.5 \times 10^{-3}$ $\mathrm{dB} / \mathrm{km}$ when $\xi=0.5$. At $F=2.58$, the $\mathrm{CL}$ accounts for $1.5 \mathrm{~dB} / \mathrm{km}$ when $\xi=0$ and $1.6 \times 10^{-4} \mathrm{~dB} / \mathrm{km}$ when $\xi=$ 0.5 . When $\xi=1$ and $\xi=2$, a further decrease, but at a slower rate, is observed in the CL values. At $F=1.66$, the $\mathrm{CL}$ is calculated as $1.1 \times 10^{-3} \mathrm{~dB} / \mathrm{km}$ when $\xi=2$. At $F=$ 2.58 , the CL is calculated as $1.0 \times 10^{-5} \mathrm{~dB} / \mathrm{km}$ when $\xi=2$. Here, it is worth-noting that having a Kagome cladding formed by more than two rings of tubes (i.e., having $\xi>2$ ) entails a marginal decrease in the $\mathrm{CL}^{24}$. In the simulations shown in Fig. 2, the core diameter $\left(D_{\text {core }}=33.5 \mu \mathrm{m}\right)$, the distance between the cladding tubes $(\delta=4.67 \mu \mathrm{m})$, the distance between the tubular and Kagome lattices $(g=$ $1.59 \mu \mathrm{m})$, the Kagome cladding pitch $(19.46 \mu \mathrm{m})$, the curvature parameter $(b=1$, which indicates circularshaped boundary arcs), and the tube and Kagome lattice thicknesses $\left(t_{\text {tubes }}=t_{\text {kago }}=1100 \mathrm{~nm}\right)$ were kept constant.

Figure 3 presents the transverse leakage maps for different $\xi$ (the fibre parameters are the same as in Fig. 2). The colour diagrams show that at $F=2.58$ and for representative values of $\xi$, the modulus of the normalised radial component of the Poynting vector, $\overline{\bar{p}}_{\mathrm{r}}=\frac{\operatorname{Re}(\vec{p} \cdot \hat{r})}{P_{\mathrm{r}}}$, where $\vec{p}=\frac{1}{2} \vec{E} \times \overrightarrow{H^{*}}$ is the Poynting vector (with $\vec{E}$ and $\vec{H}$ representing the electric and magnetic fields, respectively), $\hat{r}$ is the radial unit vector, and $P_{\mathrm{r}}=\oint \vec{p} \cdot \hat{r} \mathrm{~d} l$ is the leaked power per unit length. Indeed, $\overline{\bar{p}}_{\mathrm{r}}$ has been proved to be a useful tool in examining the leakage dynamics in IC fibres ${ }^{7}$. In the Supplemental Material, we expatiate the relationship between $\overline{\bar{p}}_{\mathrm{r}}$ and the fibre $\mathrm{CL}$ and provide a further discussion on the normalisation procedure. Additionally, we show in Fig. 3 the attenuation values calculated as $\frac{P_{\mathrm{r}}}{P_{\mathrm{m}}}$, where $P_{\mathrm{m}}$ is the real part of the mode power, with $P_{\mathrm{m}}=\operatorname{Re}\left(\iint_{\mathrm{S}_{\infty}} \vec{p} \cdot \hat{z} \mathrm{~d} S\right)$.

Consistent with the results published by Debord et al. ${ }^{7}$, the distance between the cladding tubes in the fibre design studied here $(\delta=4.67 \mu \mathrm{m})$ entails the main channel for the core fundamental mode leakage to be the direction through the lattice tubes (instead of the direction through the gap between the cladding tubes; see the results for $\xi=$ $0)$. For $\xi>0$, one sees that the presence of the Kagome lattice around the tubular lattice minimises the power flux through this leaking channel of the tubular lattice structure. This leakage reduction increases with increasing $\xi$ for the values considered here. It is noteworthy that the main leaking channels for the Kagome lattice are azimuthally shifted from the direction through the inner lattice tubes. Additionally, the vortex-like dynamics of the radial component of the Poynting vector contour lines inside the fibre structure are remarkable. This interesting behaviour, also observed in other works ${ }^{25,26}$, calls for further exploration of the transverse energy flow in HCPCFs.

Figure 4 presents the HKT lattice design potential for offering excellent $\mathrm{CL}$ and HOM extinction levels among the representative IC-HCPCF designs explored in the literature. Figure 4a shows a comparative plot between the CL of the fundamental mode in the SR-TL HCPCF (I, green line), nested (II, pink dashed line), straight-bar (III, orange dashed line), nested-rod (IV, purple dashed line), conjoined tubes $(\mathrm{V}$, grey dashed line), and hybrid design proposed herein (VI). The data represent fibres with a core diameter of $30.5 \mu \mathrm{m}$ and lattice tubes with an outer diameter and thickness of $22.1 \mu \mathrm{m}$ and $1.1 \mu \mathrm{m}$, 


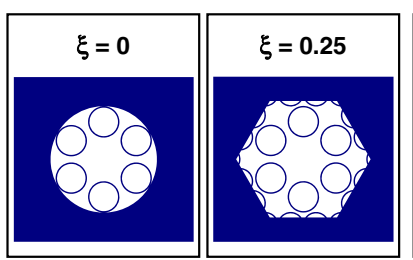

a

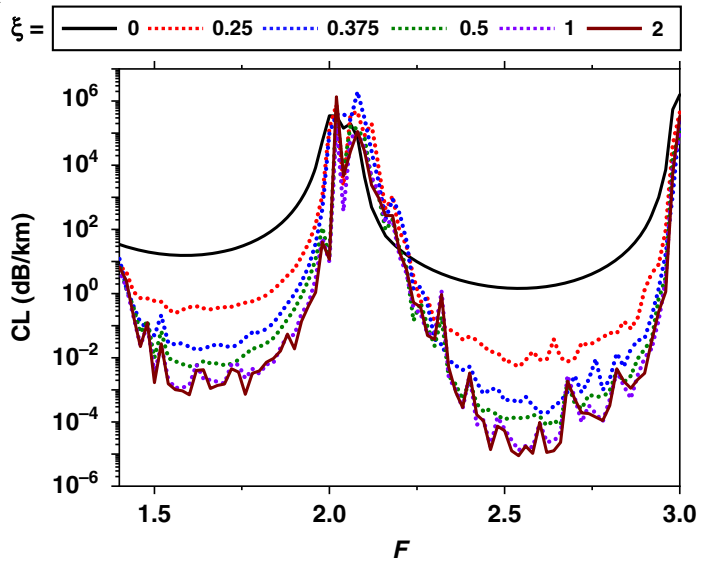

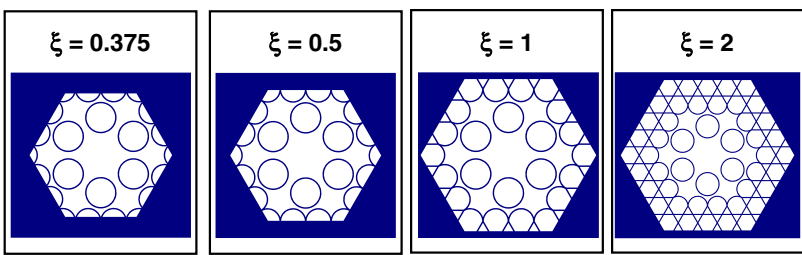

b

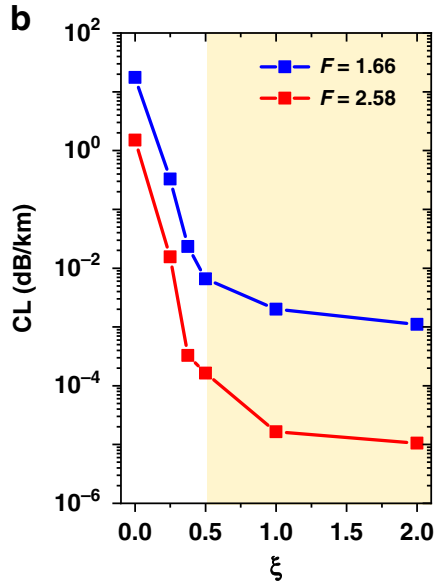

Fig. 2 Study on the effect of adding the Kagome lattice around the tubular lattice in the HKT-HCPCF design. a CL simulation results for fibre designs with different $\xi$ values (see text for the definition). $\mathbf{b} C L$ values for two selected normalised frequency (F) values

respectively. In design $\mathrm{V}$, the tubes in the second ring layer have a diameter of $26.4 \mu \mathrm{m}$, and in designs II and IV, the nested tubes have a diameter of $12.17 \mu \mathrm{m}$. The Kagome lattice pitch in designs VI and VII is set as $18 \mu \mathrm{m}$. These parameters are considered to allow a direct comparison to the data recently published by Habib et al. ${ }^{27}$.

Figure 4a shows that fibre designs II, III, IV, and V allow decreasing the CL values of SR-TL HCPCFs (I) by approximately two orders of magnitude. However, their CL remains within the range of $\sim 1-3 \mathrm{~dB} / \mathrm{km}$, with the nested design (II) having the minimum value of $0.5 \mathrm{~dB} / \mathrm{km}$ for the fibre parameters considered here. In contrast, the ideal hybrid fibre structure (VI, red line) offers a dramatic $\mathrm{CL}$ reduction. The $\mathrm{CL}$ values drop to $7 \times 10^{-5} \mathrm{~dB} / \mathrm{km}$ around $1410 \mathrm{~nm}$. In this simulation, the distance between the tubular and Kagome lattices was set as $2.04 \mu \mathrm{m}$.

While the ideal HKT-HCPCF design has suspended inner cladding tubes (therefore, it is not feasible), it is a structure of important academic interest for demonstrating the concept of the association of two IC claddings towards the reduction in the $\mathrm{CL}$, as well as the potential of the HKT-HCPCF to provide impressive low-loss figures. Taking this into account, we show in Fig. 4 (VII, blue line) a realisable version of the HKT-HCPCF. In this fibre design, the cladding tubes are connected to the Kagome lattice via the utilisation of thin tubes. Indeed, simulations show that the thinner the connection tubes are, the lower the CL values will be. Here, we choose connecting tubes with a thickness of $640 \mathrm{~nm}$ (i.e., $58 \%$ of the cladding struts and tube thickness) as a reasonable value considering the HKTHCPCF fabrication. The results show that while the addition of the connecting tubes causes the CL to increase, the minimum CL of $1.46 \times 10^{-2} \mathrm{~dB} / \mathrm{km}$ at approximately $1340 \mathrm{~nm}$ is 36 times lower than the lowest CL figure achieved with the other fibre designs between 1260 and $1400 \mathrm{~nm}$-as emphasised by the black arrow in Fig. 4a.

Additionally, we evaluate in Fig. $4 \mathrm{~b}$ the ratio between the $\mathrm{CL}$ of $\mathrm{LP}_{11}$-like modes and the $\mathrm{CL}$ of the fundamental mode $\left(\alpha_{L P 11} / \alpha_{L P 01}\right)$ to assess the potential of the fibre designs for single-mode operation. We distinguish two design groups: the first group (I, V, VI, and VII) with $\alpha_{\mathrm{LP11}} / \alpha_{\mathrm{LP01}}$ in the range of $3 \times 10^{2}$ to $3 \times 10^{4}$ and the second group (II, III, and IV) with a much lower ratio, in the range of $2-10$. This difference is readily explained by the fibre design $\mathrm{LP}_{11}$ mode resonant filtering capability ${ }^{16-18}$. Here, the hybrid fibre design is the only one that attains a better compromise in the binomial $\mathrm{CL}$ and HOM extinction. Considering the results for the hybrid fibre design, if one assumes a situation in which $99 \%$ of the power is coupled to the fundamental mode and $1 \%$ is coupled to the $\mathrm{LP}_{11}$ mode at the fibre input, an $\mathrm{HOM}$ extinction ratio higher than $40 \mathrm{~dB}$ can be estimated for a $10 \mathrm{~m}$ long fibre.

\section{Fibre fabrication and loss measurement}

Due to the potential of the HKT-HCPCF shown by the simulations, we endeavoured to obtain such a fibre experimentally. The cross-section of the fabricated fibre is presented in Fig. 5a, which was obtained by using the 


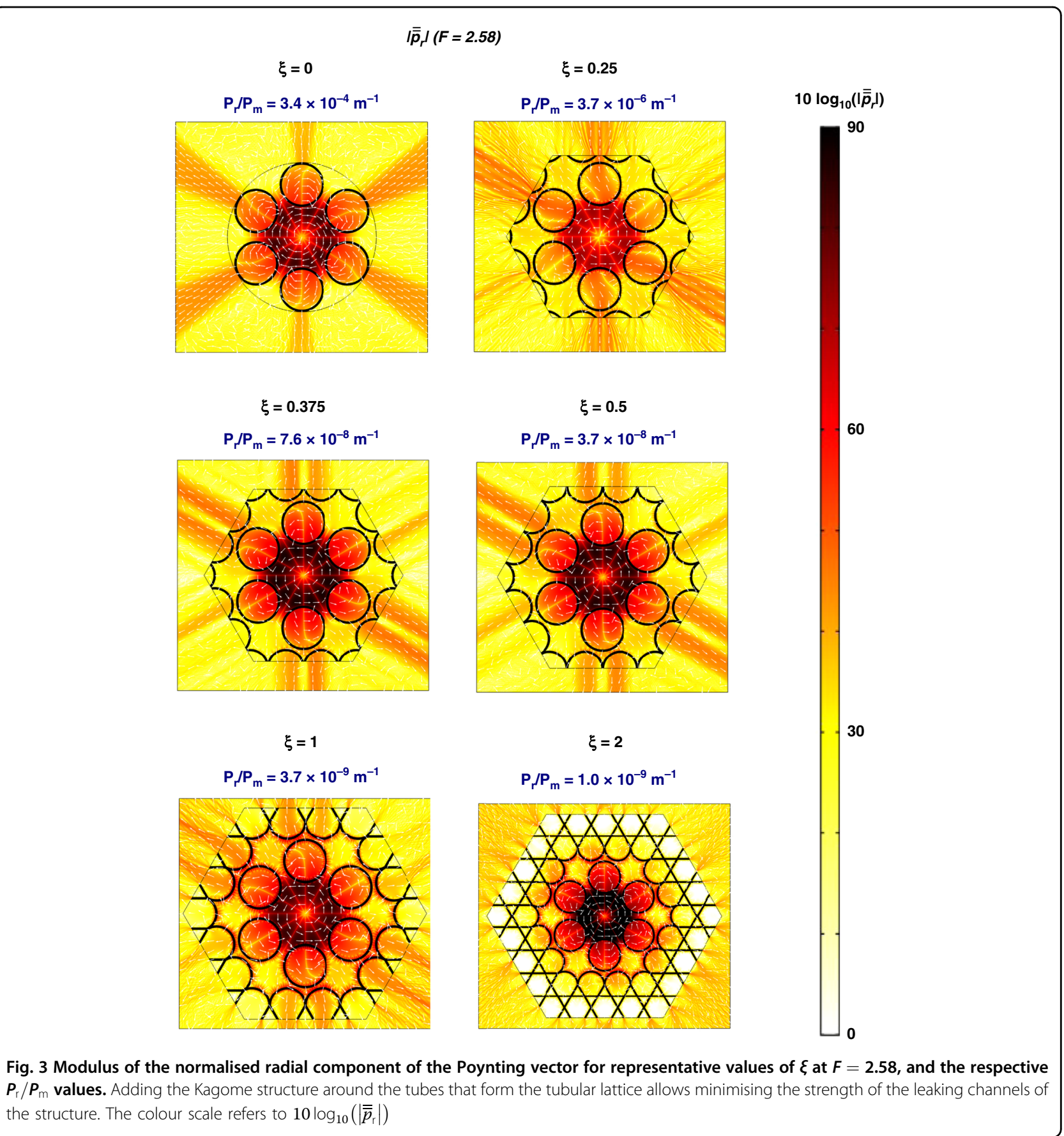

stack-and-draw technique. The tubes that form the tubular lattice have a thickness of $1.27 \mu \mathrm{m}$ and an inner diameter of $23.0 \mu \mathrm{m}$. The fibre core has a $37.1 \mu \mathrm{m}$ diameter. During fabrication, the tubes and core sizes were optimised to achieve $D_{\text {tubes }} / D_{\text {core }}=0.62$. This value is close to the optimum value of $D_{\text {tubes }} / D_{\text {core }}=0.68$, which is calculated to be the one that provides the optimum coupling between the $\mathrm{LP}_{11}$ mode guided in the core and the fundamental mode guided in the lattice tubes ${ }^{18}$. The Kagome lattice struts have a uniform thickness of $720 \mathrm{~nm}$, and the supporting tubes have a thickness of $370 \mathrm{~nm}$, both within a $50 \mathrm{~nm}$ variation (see Fig. $5 \mathrm{~b}$ for an enlarged view of the supporting tube region). In the current development stage, the fabricated fibre lengths are typically between 100 and $200 \mathrm{~m}$.

Figure 5c presents the loss of the HKT-HCPCF (blue curve), which was measured from a cutback measurement using 120 and $4 \mathrm{~m}$ long fibre pieces. A minimum loss value of $1.6 \pm 0.4 \mathrm{~dB} / \mathrm{km}$ was measured at $1050 \mathrm{~nm}$. Additionally, the simulated CL and TL (total loss) of the 

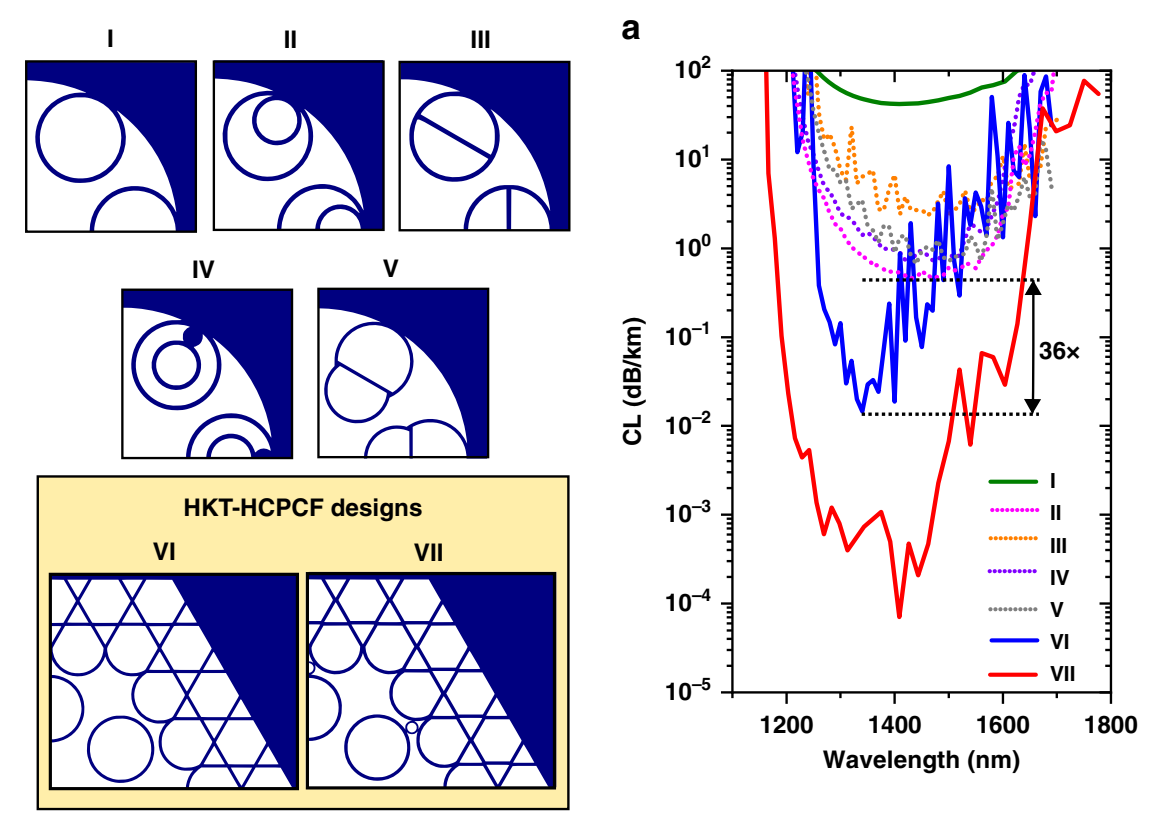

b

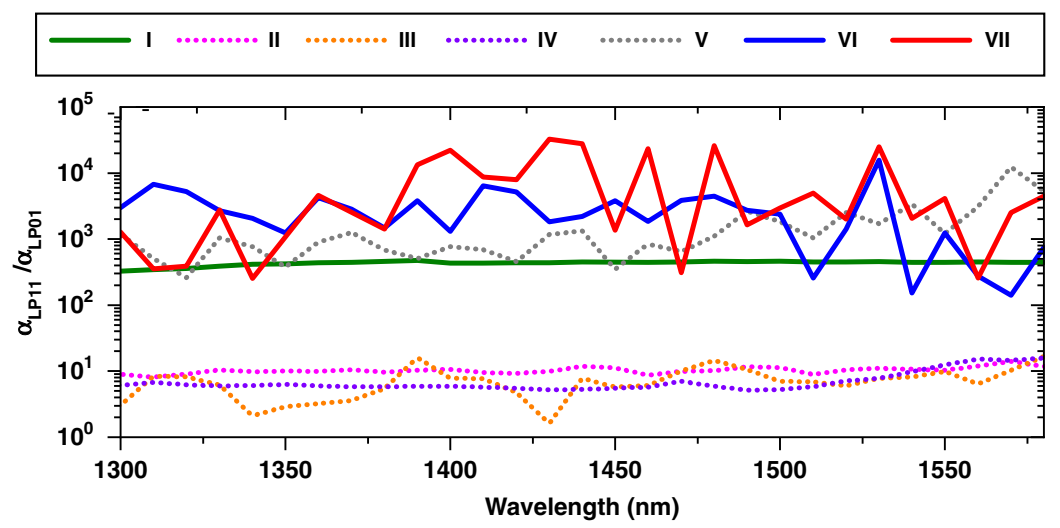

Fig. 4 Hybrid Kagome-tubular HCPCF versus reported HCPCF designs. a Fundamental mode CL simulation results for the (I) tubular, (II) nested tubular, (III) straight-bar tubular, (IV) nested-rod tubular, (V) conjoined tubes, (VI) ideal hybrid and (VII) hybrid with supporting tube fibre designs. b The ratio between the $L P_{11}$ mode and fundamental mode $C L$ values $\left(a_{L P 11} / a_{L P 01}\right)$ in the studied fibre designs

fundamental mode (green dotted curve for the CL and red dotted curve for the TL) and the TL of the representative $\mathrm{HOM}$ (namely, the $\mathrm{LP}_{11}$-like and $\mathrm{LP}_{21}$-like modes-purple and pink dotted lines, respectively) are shown in Fig. 5c. Here, it is worth clarifying that the $\mathrm{CL}$ values were calculated by considering the real fibre cross-section. In turn, the TL was calculated by using $\mathrm{TL}=\mathrm{CL}+\mathrm{SSL}$, where SSL is the surface scattering loss, which was estimated by using SSL $=\eta F_{\mathrm{cc}}\left(\frac{\lambda_{0}}{\lambda}\right)^{3}$, where $\eta$ is a constant linked to the surface roughness height, $F_{\mathrm{cc}}$ is the core mode overlap with the core contour, and $\lambda_{0}$ is a constant that allows calibrating the SSL formula ${ }^{28}$ (in Fig. 5c, $\eta=$ $0.25 \times 10^{-2}$ and $\lambda_{0}=1700 \mathrm{~nm}$ ). Good agreement is seen between the simulated TL of the fundamental mode and the experimentally measured loss. The HOM loss is found to be approximately two orders of magnitude higher than the loss of the fundamental mode, in good agreement with the predicted losses (see Fig. 4b).

\section{Modal content measurement and further characterisation results}

$S^{2}$ measurements ${ }^{21}$ were performed to quantitatively account for the modal content of the fibre. Figure $6 \mathrm{a}$ shows the $S^{2}$ trace for a $10 \mathrm{~m}$ long HKT-HCPCF. The latter shows that no contribution of the $\mathrm{LP}_{11}$ mode was detected (which shows that the 6-tube tubular-lattice fibre design filtered it). A contribution of an $\mathrm{LP}_{21}$-like mode was detected with an MPI (multipath interference) value as low as $-47.0 \mathrm{~dB}$. Table 1 shows the HOM suppression values accounted for by the $S^{2}$ measurements in the ICHCPCFs. The values reported for the tubular, nested tube, and conjoined tube designs range from 27 to $22.4 \mathrm{~dB}$ for 


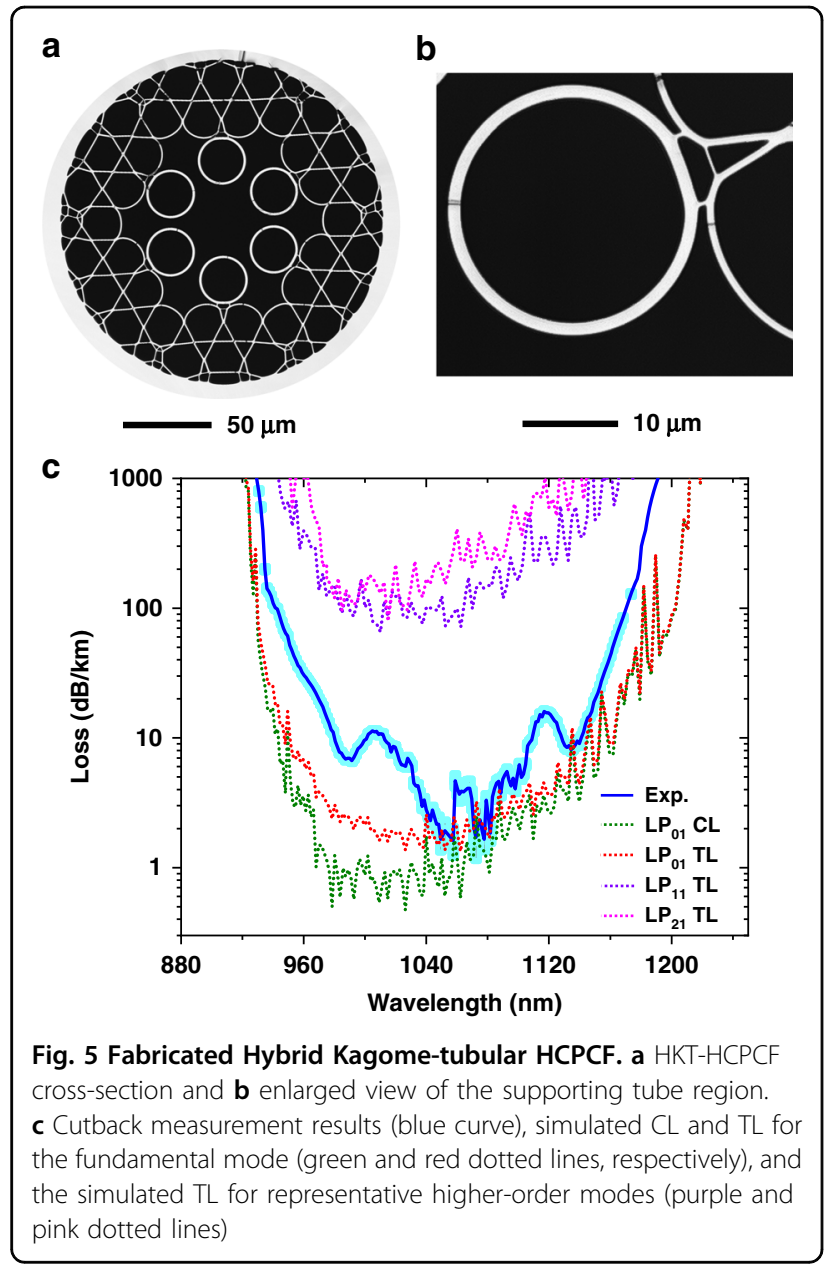

fibres with lengths between 10 and $15 \mathrm{~m}$. The HKTHCPCF reported herein, therefore, allows obtaining an improved effective single-mode operation in IC-HCPCFs.

To show the fibre single-mode operation robustness, we conducted $S^{2}$ measurements by applying an offset in the input fibre position relative to the laser beam (for a fibre with a length of $10 \mathrm{~m}$ ). The MPI values for the $\mathrm{LP}_{21}$ mode are plotted in Fig. $6 \mathrm{~b}$ as a function of the input fibre offset (the zero offset represents the optimum light transmission). It is observed that when the coupling efficiency to the $\mathrm{LP}_{21}$ mode grows as the input fibre offset becomes larger, the MPI values for the $\mathrm{LP}_{21}$ mode increase from -47.0 to $-24.7 \mathrm{~dB}$ when the input fibre offset is increased from 0 to $10 \mu \mathrm{m}$. It is noteworthy that even for this large offset in the fibre input position, the HOM contributions have a maximum MPI value of $-24.7 \mathrm{~dB}$ in the proposed HKT-HCPCF and that no $\mathrm{LP}_{11}$ contribution can be observed. Furthermore, Fig. 6c presents a typical $M^{2}$ measurement (performed at $1030 \mathrm{~nm}$ using the ISO standard, Metrolux LPM 200) for the HKTHCPCF. The $M^{2}$ values were measured to be 1.01 and 1.02 for the $x$-axes and $y$-axes, respectively. Moreover, the

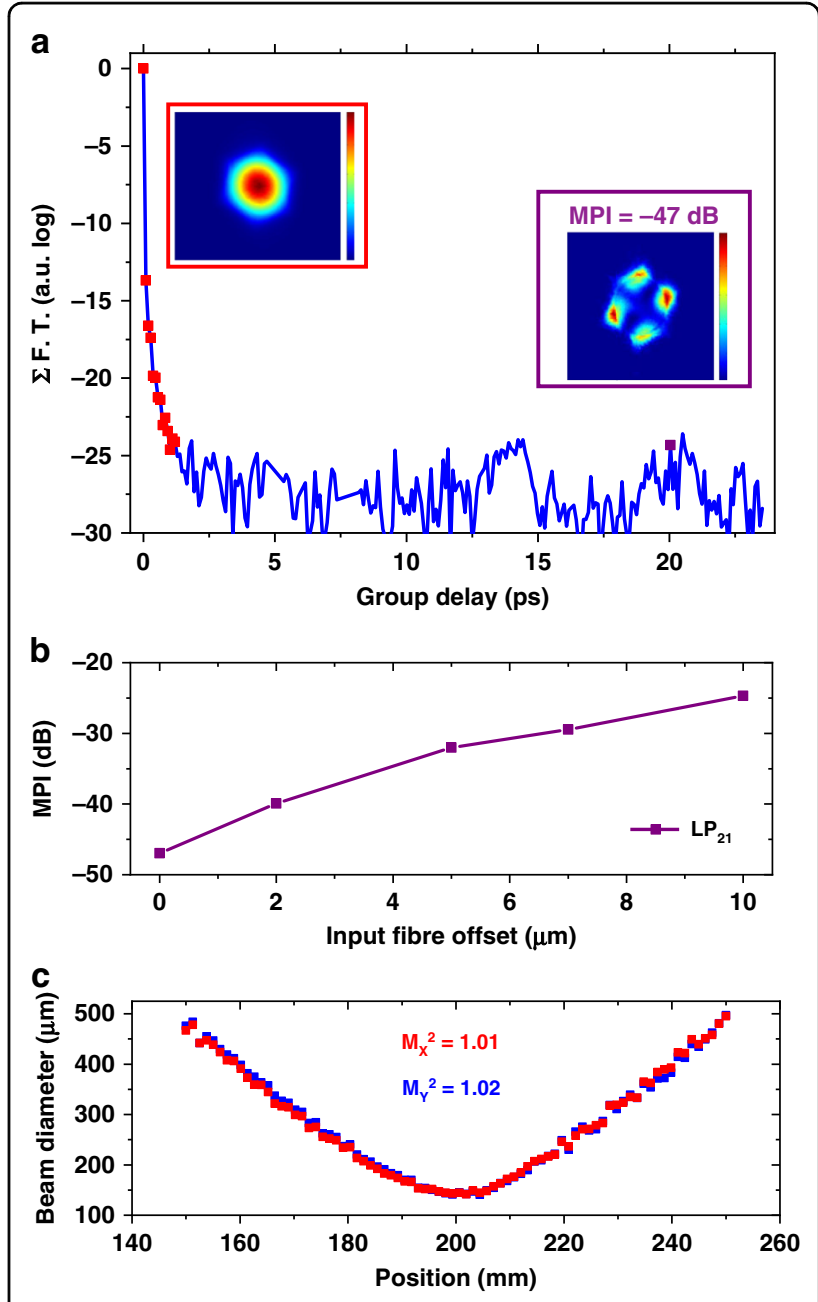

Fig. 6 HKT-HCPCF modal content characterisation. a $S^{2}$

measurement results for a $10 \mathrm{~m}$ long fibre. $\mathbf{b} \mathrm{MPI}$ as a function of the input fibre offset. c $M^{2}$ measurement results

fibre PER was tested. A maximum value of $21 \mathrm{~dB}$ was measured for a $10 \mathrm{~m}$ long fibre at $1030 \mathrm{~nm}$. Additionally, the fibre bend loss was characterised. The measurements showed that at $1100 \mathrm{~nm}$, the bend loss values were approximately $0.06 \mathrm{~dB} /$ turn when the curvature radius was $20 \mathrm{~cm}$.

Finally, the robustness of the fibre single-mode operation against laser beam misalignment with the fibre core was tested by examining the reconstructed near-field profile under different coupling conditions. To do this, we used a laser at $1064 \mathrm{~nm}$ and measured the power and the output near the field profile as the input fibre position was scanned along the horizontal and vertical axes. The scan spans $10 \mu \mathrm{m}$ along both axes, i.e., $\Delta x=\Delta y= \pm 5 \mu \mathrm{m}$. The origin corresponds to the output power maximum, measured to be $90 \%$ of the input power. For each displacement, the fibre output power and near-field profile were recorded. Figure 7a shows a colour map of the normalised 

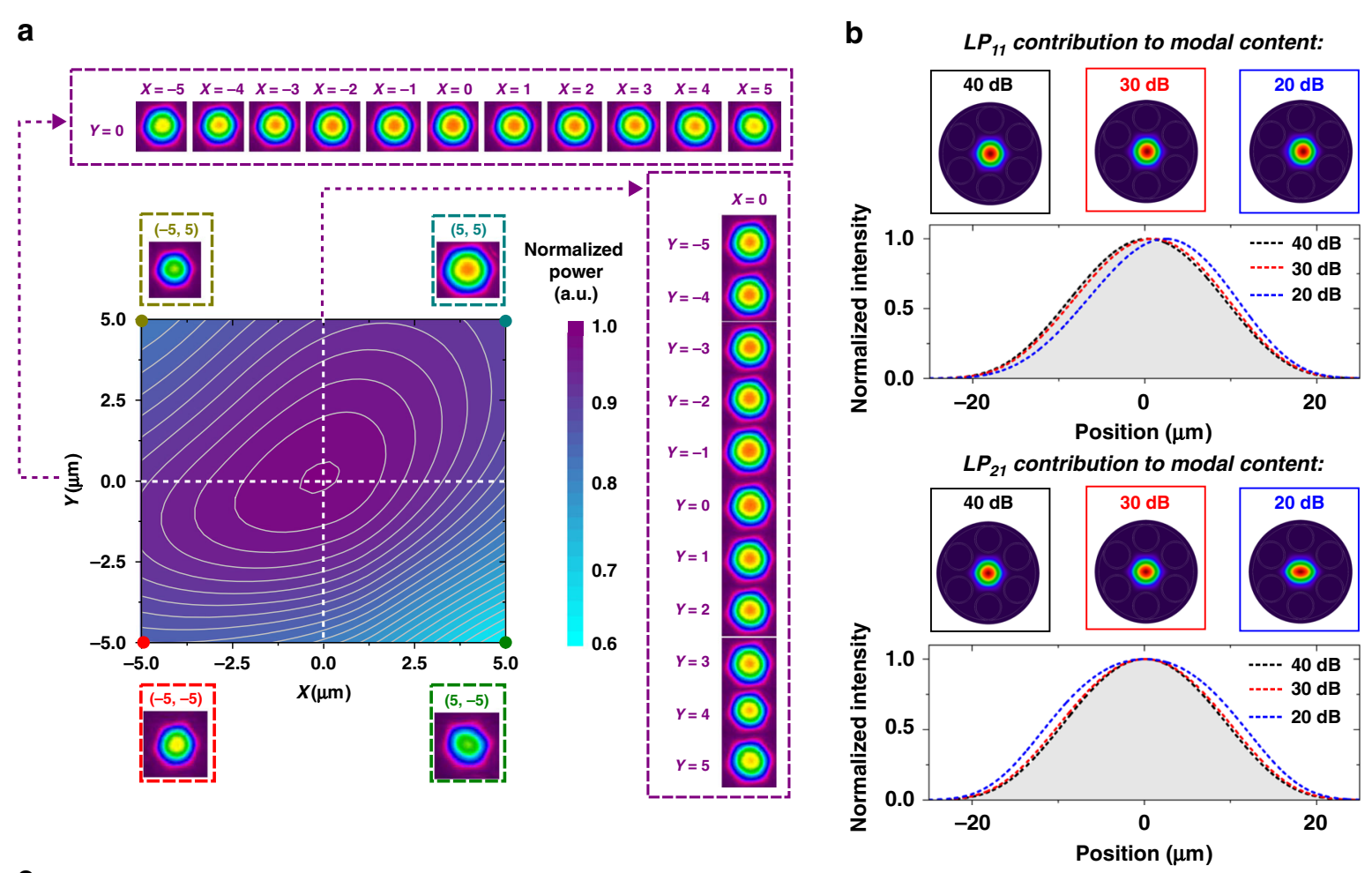

C
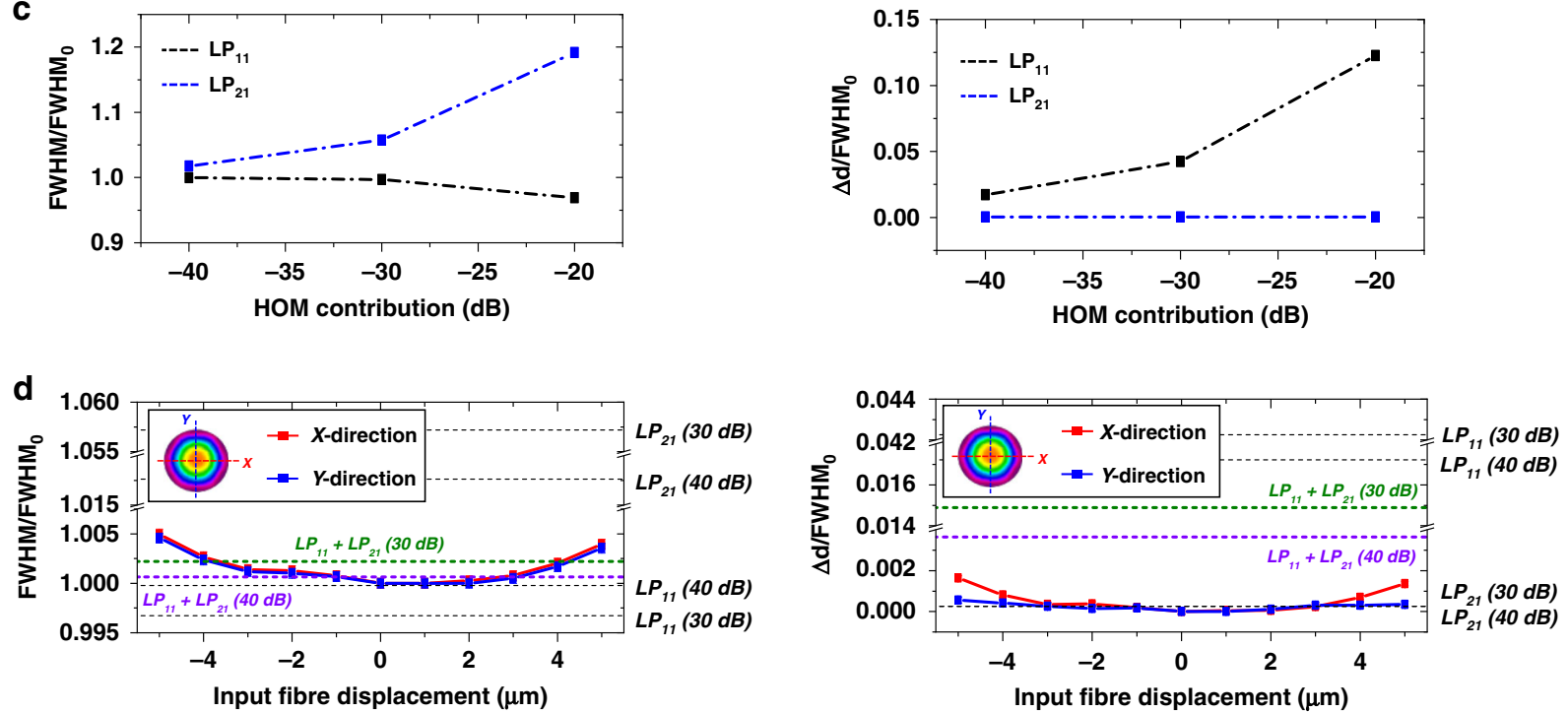

Fig. 7 Single modedness robustness. a Normalised power and near-field profiles at the fibre output for different input conditions at $1064 \mathrm{~nm}$. b Intensity mode profiles considering a fibre modal content dominated by the fundamental mode and with different HOM levels added to the modal content. c Simulated normalised beam full width at half maximum (FWHM/FHWM $M_{0}$ ) and centroid variation $\left(\triangle d / F H W M_{0}\right)$ for different $H O M$ contributions to the fibre modal content. $\mathbf{d}$ Measured FWHM/FHWM and $\triangle d / F H W M_{0}$ of the output beam as the input fibre position was scanned along the horizontal direction, i.e., $Y=0$ in a. Horizontal lines represent the simulated FWHM/FHWM $M_{0}$ and $\triangle d / F H W M_{0}$ values. In the superposition cases, we assume equally weighted combinations of the $L P_{11}$ and $L P_{21}$ modes contributing to the modal content at extents of 30 and $40 \mathrm{~dB}$

power distribution, with the selected near-field profiles shown in the insets (along the horizontal axis, along the vertical axis and at extreme positions). It is shown that over the $10 \times 10 \mu \mathrm{m}^{2}$ area, the near-field profile remained fundamental-like, and the transmitted power remained higher than $60 \%$ of the maximum. It is worthnoting that the colour map is not perfectly symmetric due to imperfect light coupling at the fibre input.

As additional indicators of the fibre effective single-mode operation, we investigate in Fig. 7 the effect of the HOM 
contribution to the full width at half maximum (FWHM) and the centroid position of the output beam. Figure $7 \mathrm{~b}$ shows the calculated intensity mode profiles, dominated by the fundamental mode, when assuming different intensity contributions from the $\mathrm{LP}_{11}$ and $\mathrm{LP}_{21}$ modes to the modal content (namely, 40, 30, and $20 \mathrm{~dB}$ ). The plots in Fig. $7 \mathrm{~b}$ present the intensity profiles along the horizontal axis for the cases mentioned above (the shaded areas represent the intensity profile of the fundamental mode). The most pronounced effect of adding the $\mathrm{LP}_{11}$ mode to the fibre modal content is shifting the beam centroid. On the other hand, the most noticeable effect of including the $\mathrm{LP}_{21}$ mode on the fibre modal content is changing the beam FWHM. These observations can be verified in Fig. 7c, where the normalised FWHM (FWHM/FHWM ${ }_{0}$, where FWHM $\mathrm{FH}_{0}$ is the full width at half maximum of the fundamental mode) and the centroid shift normalised by the $\mathrm{FHWM}_{0}(\Delta d /$ $\mathrm{FHWM}_{0}$ ) of the beams are plotted as a function of the HOM contribution to the modal content. The results show that the $\mathrm{LP}_{11}$ and $\mathrm{LP}_{21}$ contributions corresponding to a power extinction of $30 \mathrm{~dB}$ led to an $\sim 5 \%$ relative change in the FWHM and centroid position. Figure $7 \mathrm{~d}$ presents graphs of the normalised FWHM and centroid variation of the output beam as the HKT-HCPCF input position was scanned along the $X$-axis $(Y=0$ in Fig. 7a). The results show the measured FWHM/FHWM ${ }_{0}$ and $\Delta d / \mathrm{FHWM}_{0}$ to be less than $0.5 \%$ and less than $0.2 \%$, respectively, over the whole scan range. Additionally, the figure shows the HOM contribution to the FWHM/FHWM ${ }_{0}$ and $\Delta d / \mathrm{FHWM}_{0}$ variations for different $\mathrm{LP}_{11}$ and $\mathrm{LP}_{21}$ modal combinations (dashed horizontal lines). The results show that, independent of the $\mathrm{LP}_{11}$ and $\mathrm{LP}_{21}$ combination, the HOM contribution to the modal content remained less than $30 \mathrm{~dB}$ over the whole scanned area. Finally, it is noteworthy that this range in the FWHM/FHWM ${ }_{0}$ and $\Delta d / \mathrm{FHWM}_{0}$ variation was not increased when the fibre was coiled and shaken during the recording.

\section{Discussion}

The results reported herein demonstrate that the association of two IC claddings can significantly reduce the CL and modal content in HCPCFs. Specifically, we used an inner cladding with six suspended tube rings to ensure single-mode operation by resonantly filtering the lowest loss HOM and an outer cladding with a Kagome lattice for $\mathrm{CL}$ enhancement. The results show that when we surround the inner tubes with a Kagome lattice without allowing them to touch (i.e., using the ideal HKT-HCPCF), a drop in CL by 5 orders of magnitude relative to a typical tubular or Kagome cladding HCPCF and to the most representative reported HCPCF alternative designs can be achieved (a minimum of $8.6 \times 10^{-6} \mathrm{~dB} / \mathrm{km}$ was obtained with the explored fibre parameters). When considering the realisable version of the HKT-HCPCF design, the simulations revealed that a minimum loss figure of $1.42 \times 10^{-2} \mathrm{~dB} / \mathrm{km}$ at approximately $1340 \mathrm{~nm}$ can be achieved.

By making use of this concept, we developed and fabricated a hybrid cladding IC-HCPCF whereby the inner and outer cladding layers are connected via smaller and thinner tubes to keep the fibre cladding modal spectrum as close as possible to the ideal cladding design. The experimental characterisation of the fibre showed a minimum loss of $1.6 \mathrm{~dB} / \mathrm{km}$ at $1050 \mathrm{~nm}$, which is the lowest loss figure obtained at $\sim 1 \mu \mathrm{m}$ with HCPCFs, and a maximum extinction ratio of $-47.0 \mathrm{~dB}$ for the higherorder modes measured in an $S^{2}$ measurement using a $10 \mathrm{~m}$ long fibre, which is the highest extinction ratio reported in HCPCFs. We found that the single-mode operation of the reported fibre is very resilient against fibre motion and laser beam misalignment. This property is particularly important for high-power laser beam delivery in laser micromachining applications.

Improvements to the current HKT-HCPCF to achieve lower transmission should consider achieving better control of the shapes and sizes of the connecting tubes between the tubular and Kagome lattices during the fibre draw. If better control of this aspect can be obtained, a transmission loss reduction by more than one order of magnitude is expected. Moreover, further modifications to the fibre design, such as replacing some of the six tubes with others exhibiting different confining powers, will allow exploration of its potential as a polarisation-maintaining waveguide. The present results will contribute to worldwide endeavours to explore IC-HCPCFs as candidates for nextgeneration long-haul optical fibres, and will deepen our knowledge of the guidance mechanisms in these fibres.

\section{Materials and methods Fibre fabrication}

The HKT-HCPCF was obtained by using the stack-anddraw technique in a two-step process. The first step involves the preform assembly and cane drawing. The second step involves drawing the canes to the fibre dimensions. During the fibre drawing procedure, independent pressurisation was applied to the Kagome lattice, tubular lattice, and core regions to attain the required geometrical sizes.

\section{Cutback measurement}

In the cutback measurements, light from a supercontinuum light source was coupled to the fibre, and an optical spectrum analyser measured the transmitted signal. For each fibre length (120 and $4 \mathrm{~m}$ ), the transmitted spectrum was measured for three independent fibre cleavages.

\section{$S^{2}$ measurement}

The $S^{2}$ measurement setup encompassed a tuneable laser with a wavelength range between 1030 and $1070 \mathrm{~nm}$ 
(10 pm resolution) and a camera with its image acquisition routine controlled by a computer. The mode profiles and the corresponding multipath interference values were calculated from the fibre output images acquired during laser wavelength sweeping ${ }^{21}$. Before the $S^{2}$ measurements, the fibre transmission was optimised to obtain the maximum transmitted power. The MPI values shown in Fig. $6 \mathrm{~b}$ were obtained from $S^{2}$ measurements made by offsetting the fibre input from its optimum position.

\section{PER measurement}

In the PER measurements, light from a laser emitting at $1030 \mathrm{~nm}$ was launched into the fibre core, and the optical power emerging from the ports of a polarisation beam splitter was measured.

\section{Acknowledgements}

This work is funded through the PIA 4F project and the region of Nouvelle Aquitaine.

\section{Author details}

${ }^{1}$ GPPMM Group, XLIM Institute, CNRS UMR 7252, University of Limoges, Limoges 87060, France. ${ }^{2}$ GLOphotonics, 123 Avenue Albert Thomas, Limoges 87060, France. ${ }^{3}$ Department of Engineering "Enzo Ferrari", University of Modena and Reggio Emilia, Modena 41125, Italy

\section{Author contributions}

F.B. initiated and directed the work. F.A., J.H.O., F.D., B.D., and F.Ge. worked on the fibre fabrication. J.H.O. and F.A. performed the fibre characterisation measurements. J.H.O. and F.B. wrote the paper. F.Gi. and L.V. performed the simulations. All the authors discussed the results and reviewed the manuscript. F.A and J.H.O. are equally contributing authors.

\section{Data availability}

The data that support the findings of this study are available from the corresponding author upon reasonable request.

\section{Conflict of interest}

The authors declare that they have no conflict of interest.

Supplementary information is available for this paper at https://doi.org/ 10.1038/s41377-020-00457-7.

Received: 10 June 2020 Revised: 11 December 2020 Accepted: 16 December 2020

Published online: 06 January 2021

\section{References}

1. Birks, T. A., Roberts, P. J., Russell, P. S. J., Atkin, D. M. \& Shepherd, T. J. Full 2-D photonic bandgaps in silica/air structures. Electron. Lett. 31, 1941-1943 (1995).

2. Okaba, S. et al. Lamb-Dicke spectroscopy of atoms in a hollow-core photonic crystal fibre. Nat. Commun. 5, 4096 (2014).

3. Balciunas, T. et al. A strong driver in single-cycle regime based on selfcompression in a kagome fiber. Nat. Commun. 6, 6117 (2015).

4. Wang, Y. Y. et al. Design and fabrication of hollow-core photonic crystal fibers for high-power ultrashort pulse transportation and pulse compression. Opt. Lett. 37, 3111-3113 (2012).
5. Debord, B., Amrani, F., Vincetti, L., Gérôme, F. \& Benabid, F. Hollow-core fiber technology: the rising of 'Gas Photonics'. Fibers 7, 16 (2019).

6. Couny, F., Benabid, F., Roberts, P. J., Light, P. S. \& Raymer, M. G. Generation and photonic guidance of multi-octave optical frequency combs. Science $\mathbf{3 1 8}$, 1118-1121 (2007).

7. Debord, B. et al. Ultralow transmission loss in inhibited-coupling guiding hollow fibers. Optica 4, 209-217 (2017).

8. Wang, Y., Couny, F., Roberts, P. J. \& Benabid, F. Low loss broadband transmission in optimized core-shape Kagome hollow-core PCF. In Conference on Lasers and Electro-Optics (OSA, Washington, 2010), paper CPDB4.

9. Wang, Y. Y., Wheeler, N. V., Couny, F., Roberts, P. J. \& Benabid, F. Low loss broadband transmission in hypocycloid-core Kagome hollow-core photonic crystal fiber. Opt. Lett. 36, 669-671 (2011).

10. Pryamikov, A. D. et al. Demonstration of a waveguide regime for a silica hollow-core microstructured optical fiber with a negative curvature of the core boundary in the spectral region >3.5 $\mu \mathrm{m}$. Opt. Express 19, 1441-1448 (2011).

11. Maurel, M. et al. Optimized inhibited-coupling Kagome fibers at Yb-Nd:Yag (8.5 dB/km) and Ti:Sa (30 dB/km) ranges. Opt. Lett. 43, 1598-1601 (2018).

12. Benabid, F., Knight, J. C., Antonopoulos, G. \& Russell, P. St. J. Stimulated Raman scattering in hydrogen-filled hollow-core photonic crystal fiber. Science $\mathbf{2 9 8}$ 399-402 (2002).

13. Chafer, M. et al. $1 \mathrm{~km}$ hollow-core fiber with loss at the silica Rayleigh limit in the green spectral region. IEEE Photon. Technol. Lett. 31, 685-688 (2019).

14. Gao, S. et al. Hollow-core conjoined-tube negative-curvature fibre with ultralow loss. Nat. Commun. 9, 2828 (2018).

15. Jasion, G. T. et al. Hollow core NANF with $0.28 \mathrm{~dB} / \mathrm{km}$ attenuation in the $\mathrm{C}$ and $\mathrm{L}$ bands. In Optical Fiber Communication Conference, paper Th4B.4 (2020).

16. Vincetti, L. \& Setti, V. Waveguiding mechanism in tube lattice fibers. Opt. Express 18, 23133-23146 (2010).

17. Bradley, T. D. et al. Optical properties of low loss $(70 \mathrm{~dB} / \mathrm{km})$ hypocycloid-core kagome hollow core photonic crystal fiber for $\mathrm{Rb}$ and $\mathrm{Cs}$ based optical applications. J. Light. Technol. 31, 2752-2755 (2013).

18. Uebel, P. et al. Broadband robustly single-mode hollow-core PCF by resonant filtering of higher-order modes. Opt. Lett. 41, 1951-1954 (2016).

19. Gao, S. et al. Nodeless hollow-core fiber for the visible spectral range. Opt. Lett. 42, 61-64 (2017).

20. Sakr, H. et al. Record low loss hollow core fiber for the $1 \mu \mathrm{m}$ region. 2019 Conference on Lasers and Electro-Optics Europe and European Quantum Electronics Conference, paper ce_5_5 (2019).

21. Nicholson, J. W., Yablon, A. D., Ramachandran, S. \& Ghalmi, S. Spatially and spectrally resolved imaging and modal content in large-mode-area fibers. Opt. Express 16, 7233-7243 (2008)

22. Tamura, Y. et al. The first $0.14-\mathrm{dB} / \mathrm{km}$ loss optical fiber and its impact on submarine transmission. J. Light. Technol. 36, 44-49 (2018).

23. Saitoh, K. \& Koshiba, M. Confinement losses in air-guiding photonic bandgap fibers. IEEE Photon. Technol. Lett. 15, 236-238 (2003).

24. Alharbi, M. et al. Hypocycloid-shaped hollow-core photonic crystal fiber Part II: cladding effect on confinement and bend loss. Opt. Express 21, 28609-28616 (2013).

25. Jasion, G. T., Richardson, D. J. \& Poletti, F. Novel antiresonant hollow core fiber design with ultralow leakage loss using transverse power flow analysis. Optical Fiber Communication Conference (OFC) 2019, paper Th3E.2.

26. Pryamikov, A., Alagashev, G., Falkovich, G. \& Turitsyn, S. Light transport and vortex-supported wave-guiding in micro-structured optical fibres. Sci. Rep. 10, 2507 (2020).

27. Habib, M. S., Antonio-Lopez, J. E., Markos, C., Schülzgen, A. \& Amzecua-Correa, R. Single-mode, low loss hollow-core anti-resonant fiber designs. Opt. Express 27, 3824-3836 (2019).

28. Poletti, F. Nested antiresonant nodeless hollow core fiber. Opt. Express 22, 23807-23828 (2014)

29. Bradley, T. D. et al. Record low-loss $1.3 \mathrm{~dB} / \mathrm{km}$ data transmitting antiresonant hollow fibre. 2018 European Conference on Optical Communication (ECOC) 1-3 (2018). 